\section{Exploiting the Binocular Head in Polarized Light Microscopy}

Walter C. McCrone, McCrone Research Institute

Having been brought up on monocular microscopes I find the omnipresent binocular systems a luxury. To support this viewpoint l'd like to suggest some benefits you may not have considered.

Because I'm used to monocular viewing I sometimes use two different oculars, say $10 \mathrm{X}$ and $25 \mathrm{X}_{1}$ in order to scan quickly to find an area of interest and then to examine the detail with higher magnification. Occasionally / use both oculars simultaneously and "concentrate" on either image to the exclusion of the other. A better way is to set the interocular distance at the extreme setting most different from your own interocular distance. By moving your head about a centimeter either way you can use either ocular.

A variation of this theme is very helpful when you are trying to find good crystals showing useful interference figures. I simply replace one ocular with a pinhole (thoughtfully supplied by the manufacturers with all polarized light microscopes - for reasons of habit only, l'm sure). The pinhole yields a small but very sharp interference figure without the Bertrand lens. With the ocular tubes set for normal binocular viewing one can observe each crystal and, superimposed on it, the corresponding interference figure. One can, by this means, scan a field of dozens of crystals in a minute or so to select a useful view. Only the crystal or area at the very field-center will contribute to the interference figure. For measurements of optic axjal angle one then inserts the Bertrand lens for normal conoscopic viewing of the crystal located by the pin-hole method.

Finally, stereo imaging is easy by changing the interocular distance a few $\mathrm{mm}$ so that each eye looks through an outer (properly stereoscopic) or inter (pseudo-stereoscopic) edge of the oculars. I can think of some other ideas but they seem a bit too far out even for me (e.g., having an analyzer over one ocular would give crossed polars with one eye to compare with the single polar view; a very tiny, about one $\mathrm{mm}$, aperture at the eyepoint of one ocular would yield annular stop dispersion staining; a tiny one $\mathrm{mm}$ opaque stop similarly placed would yield central stop dispersion staining, etc., etc.). Still, one other practical idea is to insert a light meter in one ocular tube to record light intensity of fusion preps as they are heated to detect solid-solid phase changes and melting points you observe directly through the other ocular.

\section{Site-Specific Cross-sectioning of IC Devices for Failure Analysis by SEM/TEM: \\ Specimen Preparation Challenge and Approach} Farhad Shaapur, Arizona State University

Cross-sectioning of microelectronic devices for the purpose of construction or failure analysis by SEM and/or TEM has always been considered a major challenge. The ever increasing complexity and shrinking dimensions of these devices have pushed the art and science of the related specimen preparation beyond their conventional limits. The need for SEM failure analysis of sub-micron elements of a failed device requires the capability of cross-sectioning the sample with a high spatialresolution within a specific transverse plane. An image of the device structure obtained at sufficiently high magnification from the above specimen generally reveals the defect(s) responsible for the failure. If the imaging resolution and contrast offered by an SEM prove to be inadequate for the above purpose, device structure will be inspected via TEM. Analysis of such device by TEM imposes the additional requirement of back-thinning the above specimen to electron transparency at the site of failure.

The above challenge is currently approached through two different techniques: a) manual grinding and polishing to expose the specific site

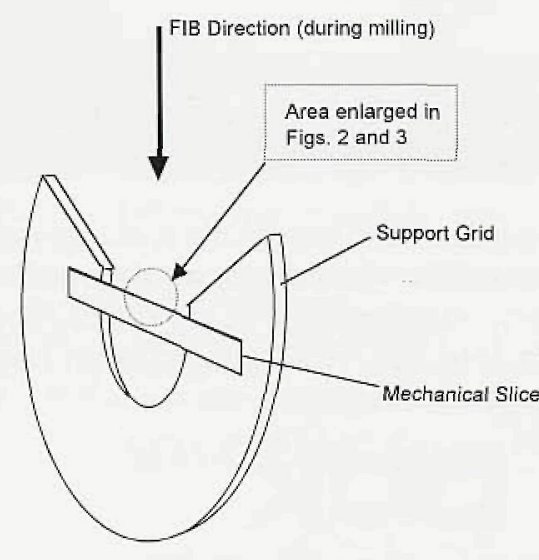

Fig. 1. Typical FIB-XTEM Specimen of interest in cross-section (for SEM analysis) followed by manual/mechanical micro-thinning from the back-side to electron transparency at the same site (for TEM analysis), or b) forming a rectangular microcrater adjacent to the site of interest using a focused ion-beam (FIB) such that a crater wall intersects and reveals the feature of interest in cross-section (for SEM analysis). lon-milling a second crater next to the first one with a side-wall intersecting the site of failure from the opposite side forms an ultra-thin electron-transparent section containing the feature of interest (for TEM analysis).

The latter technique (technique b), if used for TEM samples, also requires the following manual grinding and polishing steps prior to the FIB processing. Figure 1 schematically shows a typical FIB-milled cross-sectional TEM (FIB-XTEM) specimen. Depending on the personal preference and/or level of skill, the TEM specimen preparer may take either the "single-sided" or "double-sided" FIB milling approach. In either case, initially, he needs to prepare a thin transverse section, known as a "mechanical slice", from the device under study containing the structural feature or specific site of interest. For single-sided FIB-milling, one side of the above slice must intersect and expose the feature of interest. Subsequently, back-thinning via FIB is performed only from one side, as shown in Figure 2 . In double-sided FIB-milling, as presented in Figure 3, the mechanical slice does not expose the site of interest. Therefore, FIB-milling is required from both sides of the slice. The tool and the materials needed to accomplish above the manual preparation tasks are generally very similar or identical to those used in the former procedure (technique a).

Technique a is highly skill-based and the tool and material requirements are minimal. In contract, the FIB technique is mainly machine-based and the challenge to the specimen preparer is much more manageable. In addition, the precision offered by a FIB system unquestionably surpasses that of the manual sectioning and micro-thinning. Rather than comparing pros and cons of each technique, it is more reasonable to accept that they are complementary because each one offers some distinct and crucial capabilities to the $\mathrm{IC}$ device failure analyst.

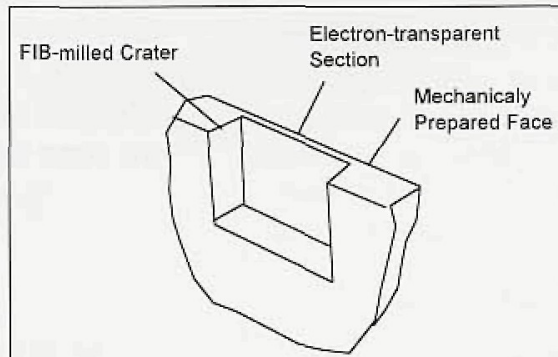

Fig.2. Single-sided FIB-XTEM Specimen

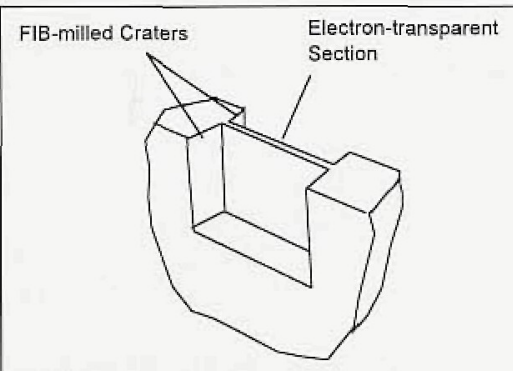

Fig.3. Double-sided FIB-XTEM Specimen 


\section{TIPS 'N HINTS}

Contributions to this new column are greatly appreciated - and may be sent to Microscopy/Microscopy Education by Fax: (413)746-6931 or by eMail: 74250.331@compuserve.com. If your Tip or Hint is used, we will send you a special "Thank You" gift from MME. -.. Barbara Foster

* Tying into the information highway: Internet. Internet is the new I communication tool of choice for microscopists. Many entries for this month's column were collected from Internet. As with any new tool, it can be confusing at first. Dr. Cesar Fermin of Tulane Medical School highly recommends The Internet Companion - A Beginner's Guide to Global Networking. Even though he has been using Internet for over two years, he finally understood the how's and why's of some of the things he had been doing. There are two ways to obtain this reference. The book is available from AddisonI Wesley Publishing Co. (ISBN 0-201-62224-6) for $\$ 11.00$ or the text can be downloaded by an anonymous FTP from FTP.STD.COM. Dr. Fermin also suggests that if you have a World Wide Web (WWM) browser like Mosaic, I the text is available free from many sources by looking in the category I "internet information". He "likes the book because it is a nice ready reference"

\ $\star$ Image Analysis in Optical Microscopy. Creating enough contrast to adequately segment one feature from another in a sample is always a challenge in image analysis. Ideally, we would like to have a bright or dark object I on a contrasting background, with good, clean edges. An easy answer: Circularly Polarized Light. Visually, the difference between this technique and regular Polarized Light Microscopy is the removal of orientation effects. In regular PLM, when a sample is rotated between crossed polars, various feaI tures go bright or dark against the black background, depending on the orientation of each refractive index with respect to the polarizer and analyzer. In $\mathrm{CPL}$, all the features which respond to polarized light appear bright against the dark background all the time, irrespective of their orientation: the ideal situation for segmentation for image analysis. CPL is quick and easy to do. First, cross the polarizer and analyzer so that the background is dark. If you I have an analyzer slot, slide in a "quarter wave plate" (lambda over 4). The \ background will turn a soft, dove gray. Next, place a matching quarter wave plate over the light port and rotate it until the background goes black again. If you don't have an analyzer slot, the first plate can go into any accessible I plane between the top of the specimen and the analyzer. Other alternatives: ask your manufacturer for a set of circularly polarized filters or buy some of the material directly from Polaroid and cut your own set. Just make sure that

the filters are in "crossed" position and that they go above and below the I sample when you but them into your microscope. - - Edifor

I * Marking Slides. Whether working in a biological or materials science Iab, getting good, legible markings on slides which will be exposed to a variety of liquids is a real challenge. Monica Nass from the Department of Pathology at Queen's University in Kingston, Ontario has had great success

\ with PAP pens. Her lab has been using these pens for several years for marking both paraffin-embedded and frozen tissue sections. Two tricks:

1. Dry the area of the slide to be marked with the pen.

2. Don't cover the section with a very large drop of liquid. If the drop is too I large, it will spill over the sides of the pen mark.

She reports that the pens, while expensive, last a very long time and

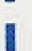
are available from Orthopath (Missisauga, Ontario).

* Formvar films. Robin Wright, at the Department of Zoology, Washington University uses dilute Formvar solutions to produce a sticky coat on grid

- bars. She reports that sections "really stay attached throughout all of the I incubations" in her immunolabeling experiments. She credits Bonnie Chojnacki with the following technique: Dip grids in a dilute Formvar solution (about $0.1 \%$ in chloroform) then dry them on filter paper. "The grids can I either be dipped individually into the Formvar and then placed on filter paper, 1 or a whole canister can be dumped into the Formvar and each grid retrieved individually onto filter paper".

$\star \quad$ If you are having trouble getting the FORMVAR films to release. Dr. Nikki Watson at the Department of Zoology, University of New England in Armidale, New South Wales sends the following solution. She first wipes a finger across her forehead and lightly coats the side of the slide on which the coating will be placed. (Hint: "works best at the end of the day"). She then suggests using a tea towel or the corner of your lab coat to buff the slide moderately clean, still leaving a microscopic layer of body grease on the surface. Proceed as usual with the Formvar coating. To release, score all around the slide edges with a sharp razor blade then just dip the slide slowly into a wide mouth vessel of distilled water so that one corner releases first. "The cast film with its grids is then very easily picked up with Parafilm rolled across the film and lifted."

* New standards for Scanning Probe Microscopy. Andy Gilicinski from Air Products and Chemicals has been keeping an eye on the emergence of new standards for this rapidly maturing technology. He reports good success with colloidal gold spheres deposited on mica, available as a commercial product from Ted Pella. He indicated that while "limited in how far up the probe they can provide information $(7,14$, and $28 \mathrm{~nm}$ ). . (they work well) for a quick and easy estimate of tip diameter and symmetry."

$\star \quad$ TEM and magnetic specimens. Joe Michael, Sandia National Laboratory, spent six years at Bethlehem Steel's research department using TEM and STEM to study steel microstructures where magnetic samples presented a real challenge. Originally, they tried to physically demagnetize the specimens but found that the fields generated within the coil usually destroyed the thin area of the specimen. His solution: make the specimen blank as thin as possible. He indicated that they usually electrochemically polished the specimen blanks to a thickness of about 50 microns before jet polishing them to perforation, minimizing the amount of magnetic material with the field of the

\section{I I

1

I

(1)

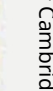

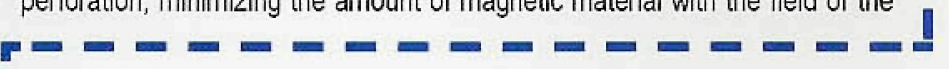

\section{New Sample Preparation Tool for Microscopy}

The new Micro Plane tools from Spectra-Tech Inc. are very useful sample preparation iterns for any microscopy laboratory. This tool allows the user to plane or scrape the surface of a solid material and produce a uniform thickness slice. An adustable screw allows the user to vary the thickness of the slice.

The carbide-steel blade version is useful for planing thin sections from most polymers, tissue samples and coated materials. The diamond-edge version produces a slice with a very clean and smooth edge. It is ideal for cutting hard polymers, multilayer materials, inorganic samples and scraping metal surfaces.

\section{- Micro Plane with Diamond-Edge Blade $\$ 495.00$ \\ - Micro Plane with Carbide-Edge Blade $\$ 425.00$}

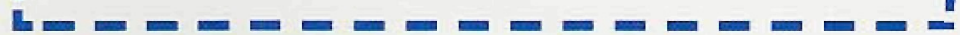

